\title{
Surgery for nonsmall cell lung cancer: can improvements be made?
}

\author{
S.G. Spiro
}

\begin{abstract}
Surgery for nonsmall cell lung cancer: can improvements be made? S.G. Spiro. (C)ERS Journals Ltd 2003.

ABSTRACT: Low-dose spiral computed tomography (CT) for the earlier detection of lung cancer is at the stage of producing hypothesis-generating studies. These studies have shown that more cancers are found at a favourable stage (IA) in prevalence screening but that the fewer numbers found in incidence screening tend to have a slightly worse stage. Randomised controlled trials will be necessary to resolve the place of spiral CT screening.

The role of neo-adjuvant chemotherapy before surgery in nonsmall cell lung cancer looks less promising than suggested by earlier studies and the place of adjuvant chemotherapy following surgery appears to be unhelpful, although results of some large, randomised international studies are still awaited.

Radical radiotherapy is a poor alternative to surgery in resectable patients who refuse or are unfit for surgery and postoperative radiotherapy is detrimental. Positron emission tomography scanning offers a genuine opportunity to identify occult disease and improve staging prior to surgery and therefore save futile thoracotomies in $\sim 20 \%$ of patients otherwise apparently suitable for resection.

Eur Respir J 2003; 21: Suppl. 39, 52s-56s.
\end{abstract}

\author{
Correspondence: S.G. Spiro \\ Consultant Physician \\ Dept of Thoracic Medicine \\ The Middlesex Hospital \\ Mortimer Street \\ London W1N 8AA \\ Fax: 442073809476 \\ E-mail: stephen.spiro@uclh.org
}

Keywords: Adjuvant

neoadjuvant chemotherapy

positron emission tomography

radical radiotherapy

Received: July 152002

Accepted after revision: August 222002
The hypothesis-generating studies using low-dose spiral computed tomography (CT) scanning in volunteer populations, particularly in the USA and Japan, have shown an up to four-fold increase in the prevalence pick-up of small nodules, usually not seen on a chest radiography, that ultimately have turned out to be malignant. These malignant small nodules are not alone and the same pilot screening studies have also picked up a large associated incidence of benign nodules on the screening scan. Much work needs to be carried out, particularly using the randomised controlled trial as the main instrument, to determine the true incidence of prevalence and annual (or regular incidence) cancers picked up by screening, and to unravel the detection of these tumours from the often plentiful benign nodules also found on these scans. Early indications, primarily based on work in the USA, suggest that the incidence of benign nodules is up to $13 \%$. This would indicate that volunteers having regular annual scans would be found to have a nodule every 5-6 yrs [1]. Despite the complexity of determining the place of spiral CT, it is to be hoped that it will have an effect on lung cancer mortality. It is only mortality that will have a bearing in screening studies, as the randomised controlled trials have to overcome the inherent statistical difficulties of lead time bias, length time bias and overdiagnosis bias. Similarly, the spiral CT is adept at identifying peripheral nodules, but is of no value or very little value at identifying cancers that originate from the central airways. The role of spiral CT as a future screening tool has still to be decided and this will not be possible until its screening value has been assessed in other parts of the world where the peripheral adenocarcinoma is less common than in the USA and Japan (e.g. Europe).

Surgical removal remains the conventional approach to lung cancer, wherever possible. The TNM (primary tumour, regional nodes, metastasis) staging classification was modified in 1997 by MounTAIN [2] and it highlights the importance of stage at presentation to prognosis. The most important stage classification in relation to screening studies is stage IA, which comprises a peripheral solitary nodule $<3 \mathrm{~cm}$ in diameter. Although the 5-yr survival for this presentation is $67 \%$ it comprises $<10 \%$ of all lung cancers and yet this is the stage of presentation that the peripheral nodule found on CT screening predominates. Whilst a review of the staging cascade shows that stage is related to prognosis, it is not perhaps so clear cut. The study by PATZ et al. [3], who reviewed a large series of stage IA (T1N0M0) lung cancers in 510 patients over $19 \mathrm{yrs}$, showed that there was no effect of tumour size on survival. This, they would argue, is due to the inherent biological properties of the cancer, which will have had $\sim 20-30$ volume doubling times of growth until it reached detection. During this period of time there would have been much opportunity to seed metastases and, therefore, tumour size itself may only be a factor in prognosis and the inherent biological aggression of the cancer itself should also be a very important factor. The staging classifications and cumulative 5-yr survival after treatment based on the pathological stage found at assessment (usually surgery) are shown 
Table 1.-Cumulative 5-yr survival after treatment based on pathological stage found at assessment

\begin{tabular}{lcccc}
\hline Stage/TNM & Subjects & \multicolumn{3}{c}{ Subjects surviving cumulative \% } \\
\cline { 3 - 5 } & & $\begin{array}{c}\text { 1 yr after } \\
\text { treatment }\end{array}$ & $\begin{array}{c}\text { 3 yrs after } \\
\text { treatment }\end{array}$ & $\begin{array}{c}5 \text { yrs after } \\
\text { treatment }\end{array}$ \\
\hline IA & & & & \\
T1N0M0 & 511 & 91 & 71 & 67 \\
IB & & & & \\
T2N0M0 & 549 & 94 & 67 & 57 \\
IIA & & & & \\
T1N1M0 & 76 & 88 & 65 & 55 \\
IIB & 375 & 77 & 47 & 39 \\
T2N1M0 & 288 & 78 & 47 & 39 \\
T3N0M0 & 87 & 76 & 47 & 38 \\
IIIA & 399 & 64 & 32 & 23 \\
T3N1M0 & 55 & 65 & 30 & 25 \\
T1-2-3N2M0 & 344 & 64 & 32 & 23 \\
\hline
\end{tabular}

TNM: primary tumour, regional nodes, metastasis staging. Modified from [2].

in tables 1 and 2. It is clear that the higher the stage, the better the patient's prognosis.

Considerable effort has been made to assess whether the addition of chemotherapy and/or radiotherapy both prior to (neo-adjuvant) or following (adjuvant) surgery can make an impact on survival. It is still not clear whether neo-adjuvant treatment can improve surgical 5-yr survival, although useful information has recently become available. The question as to whether adjuvant therapy can improve survival is also becoming clearer.

\section{Neo-adjuvant chemotherapy}

The main issue is whether neo-adjuvant chemotherapy improves survival in conventionally resectable

Table 2.-Stage grouping and TNM (primary tumour, regional nodes, metastasis) staging subset

\begin{tabular}{lc}
\hline Stage & TNM Subset \\
\hline Stage 0 & Carcinoma in situ \\
Stage IA & T1N0M0 \\
Stage IB & T2N0M0 \\
Stage IIA & T1N1M0 \\
Stage IIB & T2N1M0 \\
Stage IIIA & T3N0M0 \\
& T3N1M0 \\
& T1N2M0 \\
Stage IIIB & T2N2M0 \\
& T3N2M0 \\
& T4N0M0 \\
& T4N1M0 \\
& T4N2M0 \\
& T1N3M0 \\
Stage IV & T2N3M0 \\
& T3N3M0 \\
& T4N3M0
\end{tabular}

Reproduced with permission from [2]. patients, i.e. those with stage I or stage II disease (T1, 2 or $3 \mathrm{~N} 0$; T1, 2 or $3 \mathrm{~N} 1$ ) and also those with stage IIIA unforeseen N2 involvement found on pathological examination to contain microscopic disease. The second issue is whether chemotherapy can debulk and downstage more advanced disease, i.e. in patients who have bulky, abnormally sized ipsilateral mediastinal lymph nodes found on CT scanning that are ultimately biopsied and shown to contain tumour. These are lymph nodes $>1 \mathrm{~cm}$ in diameter in their short axis and would normally be regarded as implying unresectability. These patients would not normally be considered for surgery and would be treated by radical radiotherapy or chemotherapy-irradiation. However, if chemotherapy was truly effective, surgery might be possible if chemotherapy could downstage these patients and make them operable. It is more probable that this merely reduces the bulk of the abnormal nodes but does not "sterilise" them.

There are only three important randomised controlled-phase III studies that have assessed the role of neo-adjuvant chemotherapy in resectable patients versus surgery alone. Most of the data for neo-adjuvant chemotherapy have come from phase II trials, often where the numbers are small and the studies have not always been straightforward, i.e. some have used chemotherapy alone prior to surgery and others chemotherapy and irradiation. The phase II studies of chemotherapy followed by surgery have shown considerable activity. Response rates to chemotherapy range from $50-78 \%$. The overall peri-operative mortality ranges from $0-17 \%$, figures which extend well beyond the normal 5-8\% perioperative mortality for lobectomy and pneumonectomy in patients who have not had prior treatment. The median survival in these phase II studies has ranged from $12-20$ months and $\sim 20-30 \%$ of all these resected patients have had a subsequent local relapse [4-8].

In 1994, two small randomised controlled trials were published looking at the role of neo-adjuvant chemotherapy versus surgery alone in stage IIIA nonsmall cell lung cancer (NSCLC). There were 60 patients in each study [9-12]. These studies closed early because of a disparity in survival between the two arms in favour of neo-adjuvant chemotherapy. The studies showed marked survival differences; in the study of RoselL and co-workers [11, 12] no 5-yr survivors were found following surgery, although there was a $17 \%$ 5-yr survival rate following neoadjuvant chemotherapy and surgery. The American study of RоTH and co-workers [9, 10] found a 15\% 5 -yr survival rate following surgery and a 36\% survival rate following neo-adjuvant chemotherapy. However, the surgery arm faired particularly badly in the study of Rosell and co-workers [11, 12] and there were disparities between tumour K-ras mutation and deoxyribonucleic acid aneuploidy in the two groups which, in some studies, have been shown to be indicators of poor prognosis. These studies have made a strong case for the value of neoadjuvant chemotherapy.

Earlier this year, a much larger French study was published [13]. This study included 355 patients of 
stage I (except T1N0), stage II and stage IIIA NSCLC. Patients received primary surgery or two courses of mitomycin, ifosfamide and cisplatin combination chemotherapy followed by surgery. The patients who responded to chemotherapy had a further two courses of chemotherapy postoperatively. Any patients who were pathologically staged to have $\mathrm{T} 3$ or N2 disease in either arm then received thoracic radiotherapy. The response to treatment was high, in that $64 \%$ of patients in the neo-adjuvant arm had a chemotherapy response and $11 \%$ had a pathological complete response at surgery. However, the overall survivorship for the study was not significantly different with a median survival of 37 months for the neo-adjuvant arm and 26 months for the surgery arm alone. However, looking at subgroup analysis, there was a small, significant survival benefit for neoadjuvant chemotherapy in those patients who presented with $\mathrm{N} 0$ and $\mathrm{N} 1$ disease. There was also an increased postoperative mortality of $6.7 \%$ for the neoadjuvant arm compared to $4.5 \%$ for the surgical arm alone. The overall lethal toxicity for individuals entering the study was $7.8 \%$ compared to $6.6 \%$ in the study of RoselL and co-workers $[11,12]$. The fact that there was no additional survival advantage for patients with $\mathrm{N} 2$ disease does suggest that what advantage there may be for neo-adjuvant chemotherapy would occur in patients with very small volume disease. Although overall essentially a negative study, this study does raise the question of whether patients with stage I and stage II disease could benefit from neo-adjuvant chemotherapy. There is a current UK study open for patients with early disease who are randomised to chemotherapy or no chemotherapy prior to surgery, although patient refusal to enter the study because of the dislike of chemotherapy is slowing recruitment.

The question of whether neo-adjuvant treatment in locally advanced inoperable disease is going to be of value will be very hard to resolve. There are randomised controlled trials in progress in patients with bulky N2 disease where, following induction chemotherapy or chemo-radiotherapy, patients are randomised to surgery or completion radiotherapy or radical postoperative radiotherapy. These studies are recruiting slowly and it is very difficult to know whether this question will ever be answered, although intuitively it seems that this treatment will have very little to offer.

\section{Adjuvant chemotherapy}

The role of chemotherapy following surgery is likely to be answered over the next $2-3$ yrs, as several large groups are performing studies addressing this question. In 1995, the Nonsmall Cell Lung Cancer Collaborative Group meta-analysis [14] reported 14 trials, including 4,357 patients, where randomisation was to receive or not to receive chemotherapy following surgery. The meta-analysis showed that there was an advantage for receiving cisplatin-containing chemotherapy following surgery. The hazard ratio for the eight studies containing cisplatin regimes was
0.87 (confidence intervals $0.74-1.02$ ) and the absolute benefit for chemotherapy was $3 \%$ at 2 yrs and $5 \%$ at 5 yrs. This meta-analysis still did not produce a clearcut statistically significant advantage for adjuvant chemotherapy $(\mathrm{p}<0.8)$ and several large studies are re-addressing this question. These studies hope to recruit $>5,000$ patients in total, which should provide a sufficiently large number of subjects to obtain an answer. Recently, the Adjuvant Lung Project Italy Group have issued a final report in abstract form on their study of patients with stage I, II and IIIA NSCLC who had either no chemotherapy or three courses of mitomycin, vindesine and cisplatin following surgery [15]. The study recruited 1,209 patients between January 1994 and February 1998; 602 had chemotherapy, 594 no further treatment, $42 \%$ of patients had stage I disease, 31\% stage II and 27\% stage IIIA. Sixty-nine per cent (327 patients) completed chemotherapy but 166 underwent modifications of treatment. One hundred patients stopped chemotherapy early and 47 did not start. There were no survival differences between the two groups and there were no prognostic effects based on the distribution of p53 or K-ras markers in the population. This study is therefore negative for the value of adjuvant chemotherapy, but other studies including those from the National Cancer Institute of Canada, the North American Lung and Leukaemia Group B and the UK Big Lung Trial will be reporting their data within the next $1-2$ yrs.

In summary, neither neo-adjuvant or adjuvant chemotherapy should be given to NSCLC patients outside clinical trials.

\section{Radical radiotherapy for stage I and II disease}

There are patients who appear technically able to undergo resection, but either refuse surgery or are medically unfit. These patients historically are given radical radiotherapy with curative intent and this is common practice. However, there has only been one randomised controlled study conducted in the 1950s by the UK Medical Research Council which has assessed the value of radical radiotherapy as an alternative to resection [16]. Fifty-eight patients were randomised to resection or radical radiotherapy with survival at 4 yrs being $23 \%$ and $7 \%$, respectively. This difference was not significant as the numbers were small and only became significant when squamous cell lung cancers alone were assessed. Since then, there have been a large number of nonrandomised studies using a wide range of irradiation doses, although all these studies have suffered from the disadvantage of having no pathological stage for the patients (as they did not undergo resection or invasive staging) and therefore only have clinical staging data. Assessing these studies, the better responses to the radiotherapy radiologically was for smaller tumours, particularly those $<4 \mathrm{~cm}$ in diameter, where the complete response rate was $\sim 50 \%$ and the local relapse rate following treatment was lower than for larger tumours [16-18]. Overall, 5-yr survival in these early stage patients varied from 6 to $32 \%$ [19]. It is possible that modern 
treatment using CT planning and conformal radiotherapy, where the shape of the radiotherapy beam is moulded to the tumour, may produce better results than those quoted above, which are clearly inferior stage for stage to surgical data, but as yet there are no data to confirm or refute this.

\section{Postoperative radiotherapy}

Postoperative radiotherapy (PORT) has also been regarded as standard treatment following surgical resection where mediastinal N2 disease is found, as it can reduce local recurrence rates. However, what has remained controversial is whether improvement in local control will improve overall survival. A metaanalysis attempted to answer this question [20]. This analysis of all randomised controlled trials of surgery followed by radiotherapy or nothing included nine studies and in fact found a significant adverse effect for the addition of PORT on survival with a hazard ratio of 1.21 or a $20 \%$ increased relative risk for death. This finding has led to some considerable discussion. The results are not disputed for stage I and stage II disease but, for stage III disease, the negative impact of radiotherapy may have been due to poor field localisation, lack of CT scanning and different standards being applied in these older trials. The question, therefore, as to whether PORT has a role in stage IIIA disease following resection is doubtful and not completely resolved.

\section{Positron emission tomography scanning in staging of nonsmall cell lung cancer}

Because of the limitations of CT scanning and its high false-negative rate, particularly in the mediastinum, positron emission tomography (PET) scanning is of considerable interest. PET can detect malignancy in focal pulmonary lesions of $>1 \mathrm{~cm}$ in size with a sensitivity of $96 \%$ and a specificity of $88 \%$ [21]. Falsepositive findings in the lung are seen in granulomatous disease, rheumatoid arthritis and false-negatives can occur in carcinoid tumours and alveolar cell carcinoma as well as for small lesions $<1 \mathrm{~cm}$ in size.

PET is extremely valuable for evaluation of mediastinal lymphadenopathy. It is regarded as complementary to $\mathrm{CT}$, as $\mathrm{CT}$ provides the anatomical information PET cannot provide. A review of published studies [22] in 2001 confirmed that PET was significantly more accurate than CT for the detection of mediastinal nodal metastases with a sensitivity and specificity of 79 and $91 \%$, respectively, for PET versus 60 and $77 \%$ for CT. Studies assessing the additional value of PET in the staging of NSCLC are accumulating. The most recent study [23] randomised 188 patients to a conventional work-up versus conventional work-up and PET. They were able to exclude a greater number of patients from thoracotomy (18 of 92) compared to the group having a conventional workup alone (32 of 96). Furthermore, the number of unnecessary thoracotomies was reduced to 19 for those who had a PET scan compared to 39 in the group who did not. PET, therefore, saved unnecessary surgery in $20 \%$ of patients. PET was much better at identifying $\mathrm{N} 2$ and N3 disease and confirmed distant metastases in seven patients compared to one in the conventional group. PET was superior to CT in identifying the site for mediastinoscopy biopsy and, in another 10 patients, only the PET scan suggested that a biopsy of a particular site would prove positive. This study and others concludes that the negative predictive value of a PET scan is so high that, if an individual had a normal CT during staging and no contra-indications to thoracotomy, a PET scan should be performed and, if the mediastinum was negative on PET and there were no other contra-indications found, the patient could proceed direct to surgery without a mediastinoscopy. However, the false-positive rate for PET scans $(\sim 5 \%)$ is high enough to suggest that positive findings on a PET scan, particularly in the mediastinum, should be confirmed by mediastinoscopy [24, 25].

Positron emission tomography scanning is also of value in identifying distant metastases but in series where distant positive positron emission tomography findings are discovered, positron emission tomography can be falsely positive and a positive finding on positron emission tomography, if it were to change management, should be proven by biopsy.

\section{References}

1. Swenson SJ, Jett JR, Sloan JA, et al. Screening for lung cancer with low-dose spiral computed tomography. Am J Resp Crit Care Med 2002; 165: 508-513.

2. Mountain CF. Revisions in the International System for Staging Lung Cancer. Chest 1997; 111: 1710-1717.

3. Patz EF, Rossi S, Harpole DH, Herridone JE, Goodman PC. Correlation of tumour size and survival in patients with stage IA non-small cell lung cancer. Chest 2000; 117: 1568-1571.

4. Martini N, Kris MG, Flehinger BJ, et al. Preoperative chemotherapy for stage IIIa (N2) lung cancer: the Sloan-Kettering experience with 136 patients. Ann Thorac Surg 1993; 55: 1365-1373.

5. Burkes RL, Sheperd FA, Ginsberg RJ. Induction chemotherapy with MVP in patients with stage III (T1-3,N2M0) unresectable non-small lung cancer. The Toronto experiences. Proc Am Soc Clin Oncol 1994; 13: 327.

6. Elias AD, Skarin AT, Leong T, et al. Neoadjuvant therapy for surgically staged IIIA N2 non-small cell lung cancer (NSCLC). Lung Cancer 1997; 17: 147-161.

7. Sugarbaker DJ, Herndon J, Kohman LJ, Krasna M, Green MR. Results of cancer and leukemia group B protocol 8935. A multiinstitutional phase II trimodality trial for stage IIIA (N2) non-small-cell lung cancer. Cancer and Leukemia Group B Thoracic Surgery Group. J Thorac Cardiovasc Surg 1995; 109: 473-83; discussion 483-485.

8. de Boer RH, Smith IE, Pastorino U, et al. Preoperative chemotherapy in early stage resectable nonsmall-cell lung cancer: a randomized feasibility study justifying a multicentre phase III trial. $\mathrm{Br} J$ Cancer 1999; 79: 1514-1518.

9. Roth JA, Fossella F, Komaki R, et al. A randomized trial comparing perioperative chemotherapy and 
surgery with surgery alone in resectable stage IIIA non-small-cell lung cancer. J Natl Cancer Inst 1994; 86: 673-680.

10. Roth JA, Atkinson EN, Fossella F, et al. Long-term follow-up of patients enrolled in a randomized trial comparing perioperative chemotherapy and surgery with surgery alone in resectable stage IIIA non-smallcell lung cancer. Lung Cancer 1998; 21: 1-6.

11. Rosell R, Gomez-Codina J, Camps C, et al. A randomized trial comparing preoperative chemotherapy plus surgery with surgery alone in patients with non-smallcell lung cancer. $N$ Engl J Med 1994; 330: 153-158.

12. Rosell R, Gomez-Codina J, Camps C, et al. Preresectional chemotherapy in stage IIIA non-small-cell lung cancer: a 7-year assessment of a randomized controlled trial. Lung Cancer 1999; 26: 7-14.

13. Depierre A, Milleron B, Moro-Sibilot D, et al. Preoperative chemotherapy followed by surgery compared with primary surgery in resectable stage I (Except T1N0), II, and IIIa non-small-cell lung cancer. J Clin Oncol 2002; 20: 247-253.

14. Chemotherapy in non-small cell lung cancer: a metaanalysis using updated data on individual patients from 52 randomised clinical trials. Non-small Cell Lung Cancer Collaborative Group. BMJ 1995; 311: 899-909.

15. Tonato $\mathrm{M}$ and on behalf of the ALPI/EORTC-LCG investigators; Policlinico Hospital, Perugia, Italy. Final report of the Adjuvant Lung Project Italy (ALPI): an Italian/EORTC-LCG randomised trial of adjuvant chemotherapy in completely resected non-small cell lung cancer (NSCLC). Proc ASCO 2002; 21: 1157.

16. Morrison R, Deeley TJ, Cleland WP. The treatment of carcinoma of the bronchus: a clinical trial to compare surgery and supervoltage radiotherapy. Lancet 1963; 1: 683-684.

17. Krol AD, Aussems P, Noordijk EM, Hermans J,
Leer JW. Local irradiation alone for peripheral stage I lung cancer: could we omit the elective regional nodal irradiation? Int J Radiat Oncol Biol Phys 1996; 34: 297-302.

18. Slotman BJ, Antonisse IE, Njo KH. Limited field irradiation in early stage (T1-2N0) non-small cell lung cancer. Radiother Oncol 1996; 41: 41-44.

19. Zhang HX, Yin WB, Zhang LJ, et al. Curative radiotherapy of early operable non-small cell lung cancer. Radiother Oncol 1989; 14: 89-94.

20. Postoperative radiotherapy in non-small-cell lung cancer: systematic review and meta-analysis of individual patient data from nine randomised controlled trials. PORT Meta-analysis Trialists Group. Lancet 1998; 352: 257-263.

21. Hollings N, Shaw P. Diagnostic imaging of lung cancer. In: Spiro SG, ed. Lung Cancer. Vol. 17. Huddersfield, The Charlesworth Group, 2001; pp. 120150.

22. Dwamena BA, Sonnad SS, Angobaldo J, Wahl RL. Metastases from non-small cell lung cancer: mediastinal staging in the 1990s-meta-analytic comparison of PET and CT. Radiology 1999; 213: 530-536.

23. van Tinteren, Hoekstra OS, Smit EF, et al. Effectiveness of positron emission tomography in the preoperative assessment of patients with suspected non-small-cell lung cancer: the PLUS multicentre randomised trial. Lancet 2002; 359: 1388-1392.

24. Pieterman RM, van Putten JW, Meuzelaar JJ, et al. Preoperative staging of non-small-cell lung cancer with positron-emission tomography. $N$ Engl J Med 2000; 343: 254-261.

25. Dietlein M, Weber K, Gandjour A, et al. Costeffectiveness of FDG-PET for the management of potentially operable non-small cell lung cancer: priority for a PET-based strategy after nodal-negative CT results. Eur J Nucl Med 2002; 27: 1598-1609. 